\title{
INNER DERIVATIONS OF NON-ASSOCIATIVE ALGEBRAS
}

\author{
R. D. SCHAFER
}

In this note we propose a definition of inner derivation for nonassociative algebras. This definition coincides with the usual one for Lie algebras, and for associative algebras with no absolute right (left) divisor of zero. It is well known that all derivations of semi-simple associative or Lie algebras over a field of characteristic zero are inner.

Recent correspondence with $\mathrm{N}$. Jacobson has revealed that a number of the ideas in this note duplicate some of his current researches. ${ }^{1}$ In particular, he has shown that every derivation of a semisimple non-associative algebra (that is, direct sum of simple algebras) with a unity quantity over a field of characteristic zero is inner in this sense.

1. Preliminaries. A derivation of a non-associative algebra $\mathfrak{A}$ over a field $\mathfrak{F}$ is a linear transformation $D$ on $\mathfrak{A}$ satisfying

$$
(x y) D=x(y D)+(x D) y
$$

for all $x, y$ in $\mathfrak{A}$. It is known [2] that the set $\mathfrak{D}$ of all derivations of $\mathfrak{A}$ is a Lie algebra over $\mathfrak{F}$ if multiplication in $\mathfrak{D}$ is defined by

$$
\left[D_{1}, D_{2}\right]=D_{1} D_{2}-D_{2} D_{1}
$$

where $D_{1} D_{2}$ is the ordinary (associative) multiplication of linear transformations. $\mathfrak{D}$ is called the derivation algebra of $\mathfrak{A}$.

If we write $R_{y}$ for the right multiplication

$$
x \rightarrow x y=x R_{y} \quad \text { for all } x \text { in } \mathfrak{A}
$$

and $L_{x}$ for the left multiplication

$$
y \rightarrow x y=y L_{x}
$$

for all $y$ in $\mathfrak{A}$,

the definition (1) is seen to be equivalent to either one of

$$
\left[R_{y}, D\right]=R_{y D} \quad \text { for all } y \text { in } \mathfrak{A}
$$

or

$$
\left[L_{x}, D\right]=L_{x D} \quad \text { for all } x \text { in } \mathfrak{A} \text {. }
$$

Presented to the Society, April 30, 1949; received by the editors June 20, 1948.

$1 \mathrm{~N}$. Jacobson, Derivation algebras and multiplication algebras of semi-simple Jordan algebras, to appear in Ann. of Math.

${ }^{2}$ Numbers in brackets refer to the references cited at the end of the paper. 
We denote by $R(\mathfrak{H})$ (or $L(\mathfrak{R})$ ) the set of all right (or left) multiplications of $\mathfrak{A}$. Then (3) and (4) imply

$$
[R(\mathfrak{R}), \mathfrak{D}] \leqq R(\mathfrak{A}), \quad[L(\mathfrak{H}), \mathfrak{D}] \leqq L(\mathfrak{A}),
$$

where by $[\subseteq, \mathfrak{T}]$ we mean the linear set spanned by all $[S, T]$ for $S$ in $\mathfrak{S}, T$ in $\mathfrak{T}$. Anti-commutativity

$$
[S, T]=-[T, S]
$$

and the Jacobi identity

$$
[[S, T], U]+[[T, U], S]+[[U, S], T]=0
$$

for linear transformations on $\mathfrak{A}$ imply

$$
\begin{gathered}
{[\mathfrak{S}, \mathfrak{T}]=[\mathfrak{T}, \mathfrak{S}],} \\
{[[\mathfrak{S}, \mathfrak{T}], \mathfrak{u}] \leqq[[\mathfrak{T}, \mathfrak{u}], \mathfrak{S}]+[[\mathfrak{U}, \mathfrak{S}], \mathfrak{T}]}
\end{gathered}
$$

for linear sets $\mathfrak{S}, \mathfrak{T}, \mathfrak{U}$ of linear transformations on $\mathfrak{A} .^{3}$

A non-associative algebra $\mathfrak{A}$ which is not the zero algebra of dimension one is called simple in case its only ideals are $\{0\}$ and $\mathfrak{A}$. A semi-simple non-associative algebra $\mathfrak{A}$ is the direct sum $\mathfrak{A}=\mathfrak{A}_{1}$ $\oplus \cdots \oplus \mathfrak{A}_{s}$ of simple components $\mathfrak{A}_{i}$.

The center $\mathbb{Z}$ of a non-associative algebra $\mathfrak{A}$ consists of all elements $c$ in $\mathfrak{A}$ such that

$$
L_{c}=R_{c}, \quad R_{y} R_{c}=R_{c} R_{y}=R_{c y}
$$

for all $y$ in $\mathfrak{A}$. The center of $\mathfrak{A}$ is characteristic; that is, $\mathfrak{B D} \leqq \mathfrak{Z}$. For $L_{c D}=\left[L_{c}, D\right]=\left[R_{c}, D\right]=R_{c D}$, while $R_{y} R_{c D}=\left[R_{y} R_{c}, D\right]-\left[R_{y}, D\right] R_{c}$ $=\left[R_{c} R_{y}, D\right]-R_{c}\left[R_{y}, D\right]=\left[R_{c}, D\right] R_{y}=R_{c D} R_{y}=R_{(c y) D}-R_{c(y D)}=R_{(c D) y}$.

Also the simple components of a semi-simple algebra are characteristic, for $\mathfrak{A}_{i}^{2}=\mathfrak{A}_{i}$ implies that $x$ in $\mathfrak{A}_{i}$ may be written in the form $x=\sum y_{j} z_{j}$ for $y_{j}, z_{j}$ in $\mathfrak{A}_{i}$. Then $x D=\sum\left(y_{j} z_{j}\right) D=\sum y_{j}\left(z_{j} D\right)+\sum\left(y_{j} D\right) z_{j}$ is in $\mathfrak{A}_{i}$ since $\mathfrak{A}_{i}$ is an ideal of $\mathfrak{A}$.

The center $\mathbb{Z}$ of a simple non-associative algebra $\mathfrak{A}$ is either $\{0\}$ or a field. If $\mathfrak{A}$ contains a unity element 1 , then $\mathbb{Z} \neq\{0\}$, and $\mathfrak{A}$ may be regarded as an algebra over 3 . As such, it is central simple (that is, simple for all scalar extensions).

2. Inner derivations. Let $\mathfrak{M}$ be a linear set of transformations on $\mathfrak{A}$, and write

$$
\mathfrak{M}_{1}=\mathfrak{M}, \quad \mathfrak{M}_{i}=\left[\mathfrak{M}_{1}, \mathfrak{M}_{i-1}\right], \quad i=2,3, \cdots .
$$

${ }^{3}$ Throughout this note we use the notation $\mathfrak{B}+\mathfrak{C}$ for the sum of the linear sets $\mathfrak{B}$, $\mathfrak{E}$; we do not mean necessarily that $\mathfrak{B} \cap \mathfrak{C}=\{0\}$. 
Then the set $\mathfrak{R}=\mathfrak{M}_{1}+\cdots+\mathfrak{M}_{i}+\cdots$ is the smallest Lie algebra containing $\mathfrak{M}$. For, when we prove

$$
\left[\mathfrak{M}_{i}, \mathfrak{M}_{j}\right] \leqq \mathfrak{M}_{i+j}, \quad i, j=1,2, \cdots,
$$

we have proved that $\&$ is a Lie algebra. By virtue of $(8)$ it is sufficient to prove (12) for $j \geqq i$. If $i=1$ we have (12) by definition. We assume therefore that $\left[\mathfrak{M}_{k}, \mathfrak{M}_{l}\right] \leqq \mathfrak{M}_{k+l}$ for any $l \geqq k$ and for $k<i$. Then $\left[\mathfrak{M}_{i}, \mathfrak{M}_{j}\right]=\left[\left[\mathfrak{M}_{1}, \mathfrak{M}_{i-1}\right], \mathfrak{M}_{j}\right] \leqq\left[\left[\mathfrak{M}_{i-1}, \mathfrak{M}_{j}\right], \mathfrak{M}_{1}\right]+\left[\left[\mathfrak{M}_{j}, \mathfrak{M}_{1}\right], \mathfrak{M}_{i-1}\right]$ $\leqq\left[\mathfrak{M}_{i+j-1}, \mathfrak{M}_{1}\right]+\left[\mathfrak{M}_{j+1}, \mathfrak{M}_{i-1}\right] \leqq \mathfrak{M}_{i+j}$ by (9). Now any Lie algebra containing $\mathfrak{M}$ contains all $\mathfrak{M}_{i}$ and therefore $\mathfrak{R}$, so $\mathfrak{R}$ is the smallest Lie algebra containing $\mathfrak{M}$.

The role of $\mathfrak{R}$ is analogous to that of the enveloping algebra of $\mathfrak{M}$, which is the smallest associative algebra containing $\mathfrak{M}$. Clearly \& is contained in the enveloping algebra of $\mathfrak{M}$.

Let $\mathfrak{M}$ be the set $R(\mathfrak{A})+L(\mathfrak{A})$ spanned by the right and left multiplications of $\mathfrak{A}$. Then we call $\mathbb{R}$ the Lie transformation algebra of $\mathfrak{A} . \mathbb{R}$ is contained in the so-called transformation algebra $T(\mathfrak{H})$, the enveloping algebra of the right and left multiplications and the identity $I$.

Definition. We call a derivation $D$ of a non-associative algebra $\mathfrak{A}$ inner in case $D$ is in the Lie transformation algebra $\mathfrak{R}$ of $\mathfrak{A}$.

We recall that an algebra $\mathfrak{A}$ is associative in case

$$
(x y) z=x(y z)
$$

for $x, y, z$ in $\mathfrak{A}$.

It is easy to see that (13) is equivalent to any one of

$$
L_{x y}=L_{y} L_{x}, \quad\left[L_{x}, R_{z}\right]=0, \quad R_{y} R_{z}=R_{y z}
$$

Let

$$
D=R_{d}-L_{d}
$$

for $d$ in $\mathfrak{A}$;

then $D$ is a derivation of $\mathfrak{A}$ by (3) and (14) since $\left[R_{y}, R_{d}-L_{d}\right]$ $=\left[R_{y}, R_{d}\right]=R_{y d-d y}$. Derivations of this form have always been called inner derivations of the associative algebra $\mathfrak{A}$. They are inner derivations by our definition.

$$
\begin{aligned}
& \text { THEOREM 1. The Lie transformation algebra of an associative algebra } \\
& \mathfrak{A} \text { is } \\
& \text { (16) } \quad \mathfrak{R}=R(\mathfrak{l})+L(\mathfrak{l}) .
\end{aligned}
$$

If $\mathfrak{A}$ has no absolute right (left) divisor of zero, then a derivation $D$ of $\mathfrak{A}$ is inner if and only if $D$ has the form (15).

Any element of $R(\mathfrak{A})+L(\mathfrak{H})$ has the form $R_{x}+L_{y}$. Then (14) 
implies that $\left[R_{x_{1}}+L_{y_{1}}, R_{x_{2}}+L_{y_{2}}\right]=\left[R_{x_{1}}, R_{x_{2}}\right]+\left[L_{y_{1}}, L_{y_{2}}\right]=R_{\left[x_{1}, x_{2}\right]}$ $+L_{\left[y_{2}, y_{1}\right]}$ in $R(\mathfrak{H})+L(\mathfrak{H})$, so that $R(\mathfrak{H})+L(\mathfrak{H})$ is itself a Lie algebra, and (16) holds. Let $R_{d}+L_{f}$ in $\mathfrak{\&}$ be a derivation of $\mathfrak{A}$. Since (15) is a derivation, so is $\left(R_{d}+L_{f}\right)-\left(R_{d}-L_{d}\right)=L_{f+d}$. From (1) we have $(f+d) x y=x(f+d) y+(f+d) x y$, or $x(f+d) y=x R_{(f+d) y}=0$ for all $x, y$ in $\mathfrak{A}$. Hence $R_{(f+d) y}=0$. Assuming there is no absolute right divisor of zero in $\mathfrak{A}$, we have $(f+d) y=y L_{f+d}=0$ for all $y$, or $L_{f+d}=0, R_{d}+L_{f}$ $=R_{d}-L_{d}$. A similar argument may be made in case $\mathfrak{A}$ contains no absolute left divisor of zero.

The identities

$$
x y=-y x, \quad(x y) z+(y z) x+(z x) y=0
$$

define a Lie algebra. These together are equivalent to

$$
L_{x}=-R_{x}, \quad\left[R_{y}, R_{z}\right]=R_{y z} .
$$

Then (3) and (18) imply that

$$
D=R_{d} \quad \text { for any } d \text { in } \mathfrak{A}
$$

is a derivation of $\mathfrak{A}$; this has always been called an inner derivation of a Lie algebra. It is also inner by our definition.

THEOREM 2. The Lie transformation algebra of a Lie algebra $\mathfrak{A}$ is

$$
\mathfrak{R}=R(\mathfrak{R}) \text {. }
$$

$A$ derivation $D$ of $\mathfrak{A}$ is inner if and only if $D$ has the form (19).

For $L(\mathfrak{H})=R(\mathfrak{H})$ and $[R(\mathfrak{I}), R(\mathfrak{H})] \leqq R(\mathfrak{H})$ by $(18)$.

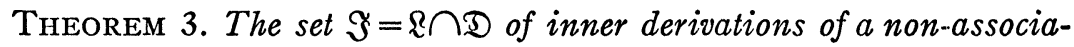
tive algebra $\mathfrak{A}$ is an ideal in the derivation algebra $\mathfrak{D}$.

If $J$ is in $\Im$ and $D$ in $\mathscr{D}$, we have $[J, D]$ in $\mathfrak{D}$. Moreover, $J$ may be written in the form $J=\sum M_{i}, M_{i}$ in $\mathfrak{M}_{i}$. Now

$$
\left[\mathfrak{M}_{i}, \mathfrak{D}\right] \leqq \mathfrak{M}_{i} \text {, }
$$

$$
i=1,2, \cdots \text {. }
$$

The case $i=1$ of (21) is given by (5). We assume (21) in a proof by induction. Since $M_{i+1}$ is a sum of elements of the form $\left[M_{1}, M_{i}\right]$, we have $\left[M_{i+1}, D\right]$ a sum of elements of the form $\left[\left[M_{1}, M_{i}\right], D\right]$ $=-\left[\left[M_{i}, D\right], M_{1}\right]-\left[\left[D, M_{1}\right], M_{i}\right]$ in $\left[\mathfrak{M}_{i}, \mathfrak{M}_{1}\right]+\left[\mathfrak{M}_{1}, \mathfrak{M}_{i}\right]=\mathfrak{M}_{i+1}$. Then $[J, D]$ is in $\sum\left[\mathfrak{M}_{i}, \mathfrak{D}\right] \leqq \sum \mathfrak{M}_{i}=\mathfrak{R}$, and $[J, D] \in \mathfrak{S}=\mathfrak{R} \cap \mathfrak{D}$.

Corollary. If $\mathfrak{D}$ is simple and $\Im \neq\{0\}$, then $\Im=\mathfrak{D}$; that is, all the derivations of $\mathfrak{A}$ are inner.

THEOREM 4. Let $\mathfrak{A}=\mathfrak{H}_{1} \oplus \cdots \oplus \mathfrak{A}_{\mathrm{s}}$ be a semi-simple non-associa- 
tive algebra, $\mathfrak{I}_{i}$ simple. All the derivations of $\mathfrak{A}$ are inner if and only if all the derivations of $\mathfrak{A}_{i}$ are inner $(i=1,2, \cdots, s)$.

Since $\mathfrak{A}_{i}$ is characteristic, any derivation $D$ of $\mathfrak{A}$ induces a derivation $D^{(i)}$ on $\mathfrak{A}_{i}$, and

$$
D=D_{1}+\cdots+D_{s}, \quad D_{i} \text { derivations of } \mathfrak{A} \text {, }
$$

where the restriction of $D_{i}$ to $\mathfrak{A}_{i}$ is $D^{(i)}$ and $\mathfrak{A}_{j} D_{i}=\{0\}$ for $j \neq 1$. Conversely, if $D^{(i)}$ is a derivation of $\mathfrak{A}_{i}$, the transformation $D_{i}$ on $\mathfrak{A}$, whose restriction to $\mathfrak{A}_{i}$ is $D^{(i)}$ and for which $\mathfrak{A}_{j} D_{i}=\{0\}$ if $j \neq i$, is a derivation of $\mathfrak{A}$; then so is the sum (22). Since the $\mathfrak{A}_{i}$ are pairwise orthogonal ideals, a similar decomposition of right and left multiplications is possible, and any element $T$ of the Lie transformation algebra $\mathfrak{R}$ of $\mathfrak{A}$ may be written as

$$
T=T_{1}+\cdots+T_{s}
$$

where $\mathfrak{A}_{j} T_{i}=\{0\}$ for $j \neq i$, and the restriction of $T_{i}$ to $\mathfrak{A}_{i}$ is an element $T^{(i)}$ of the Lie transformation algebra of $\mathfrak{H}_{i}$. Conversely if $T^{(i)}$ is an element of the Lie transformation algebra of $\mathfrak{A}_{i}$, define the transformation $T_{i}$ of $\mathfrak{A}$ whose restriction to $\mathfrak{A}_{i}$ is $T^{(i)}$ and for which $\mathfrak{A}_{j} T_{i}=\{0\}$ if $j \neq i$; then $T_{i}$ is in $\mathbb{R}$, and so is the sum (23). Then $D=T$ if and only if $D^{(i)}=T^{(i)}(i=1,2, \cdots s)$.

3. Alternative algebras. An alternative algebra $\mathfrak{A}$ is a non-associative algebra in which

$$
x^{2} y=x(x y), \quad y x^{2}=(y x) x \quad \text { for all } x, y \text { in } \mathfrak{A} .
$$

All associative algebras are alternative. Moreover, all simple alternative algebras are associative, except for algebras which are CayleyDickson algebras over their centers [4]. Cayley-Dickson algebras are algebras $(5$ of dimension 8 formed from a (generalized) quaternion algebra $\mathfrak{Q}$ as follows: the elements of $\mathfrak{S}=\mathfrak{Q}+v \mathfrak{Q}$ are $q_{1}+v q_{2}, q_{i}$ in $\mathfrak{Q}$; multiplication in $\mathfrak{S}$ is defined by

$$
\left(q_{1}+v q_{2}\right)\left(q_{3}+v q_{4}\right)=\left(q_{1} q_{3}+\gamma q_{4} \bar{q}_{2}\right)+v\left(\bar{q}_{1} q_{4}+q_{3} q_{2}\right)
$$

where $\gamma \neq 0$ in $\mathfrak{F}$ and $\bar{q}=t(q) 1-q$ for $q$ in $\mathfrak{Q}$ with $q^{2}-t(q) q+n(q) 1=0$.

In an alternative algebra $\mathfrak{U}$ the "associator" $\left[x_{1}, x_{2}, x_{3}\right]=\left(x_{1} x_{2}\right) x_{3}$ $-x_{1}\left(x_{2} x_{3}\right)$ "alternates"; that is, $\left[x_{1}, x_{2}, x_{3}\right]=\epsilon\left[x_{i_{1}}, x_{i_{2}}, x_{i_{3}}\right]$ for any permutation $i_{1}, i_{2}, i_{3}$ of $1,2,3$, where $\epsilon$ is 1 in case the permutation is even, -1 in case it is odd. Equivalently,

$$
\begin{aligned}
R_{x z}-R_{x} R_{z} & =R_{z} R_{x}-R_{z x}=L_{z x}-L_{x} L_{z}=L_{z} L_{x}-L_{x z} \\
& =\left[L_{x}, R_{z}\right]=\left[R_{x}, L_{z}\right] .
\end{aligned}
$$


Now (26) implies that

$$
\left[R_{x}, R_{z}\right]=R_{[x, z]}-2\left[L_{x}, R_{z}\right]
$$

and

$$
\left[L_{x}, L_{z}\right]=-L_{[x, z]}-2\left[L_{x}, R_{z}\right],
$$

where by $[x, z]$ we mean $x z-z x$ in $\mathfrak{A}$. Also

$$
D_{1}=3\left[L_{x}, L_{z}\right]+2 R_{[x, z]}+L_{[x, z]}
$$

is a derivation of $\mathfrak{A}$ for any $x, z$ in $\mathfrak{A}$, for it is easy to see from (26) that

$$
\left[L_{y},\left[L_{x}, L_{z}\right]\right]=L_{y\left[L_{x}, L_{z}\right]+y[x, z]}
$$

while (28) gives $\left[L_{y}, 2 R_{[x, z]}+L_{[x, z]}\right]=-L_{[y,[x, z]]}$. With (27) and (28) it is easy to write several variants of (29); indeed, if the characteristic of $\mathfrak{F}$ is not two, we obtain the symmetrical expression

$$
D=\left[L_{x}, L_{z}\right]+\left[L_{x}, R_{z}\right]+\left[R_{x}, R_{z}\right]
$$

for $D=D_{1} / 2$ in (29). Then the sum

$$
D=\sum_{i}\left(\left[L_{x_{i}}, L_{z_{i}}\right]+\left[L_{x_{i}}, R_{z_{i}}\right]+\left[R_{x_{i}}, R_{z_{i}}\right]\right)
$$

is also a derivation of $\mathfrak{A}$ for $x_{i}, z_{i}$ in $\mathfrak{A}$.

THEOREM 5. The Lie transformation algebra of an alternative algebra $\mathfrak{A}$ over $\mathfrak{F}$ of characteristic not two is

$$
\mathfrak{R}=R(\mathfrak{H})+L(\mathfrak{U})+[L(\mathfrak{H}), R(\mathfrak{H})] .
$$

Let $\mathfrak{M}_{1}=R(\mathfrak{R})+L(\mathfrak{R}) \quad$ and $\quad \mathfrak{R}_{0}=\mathfrak{M}_{1}+[L(\mathfrak{H}), \quad R(\mathfrak{U})]$. Then $\mathfrak{M}_{2}$ $=\left[\mathfrak{M}_{1}, \mathfrak{M}_{1}\right] \leqq \mathfrak{R}_{0}$ by $(27)$ and (28). Assume $\mathfrak{M}_{i-1} \leqq \mathfrak{R}_{0}$. Then $\mathfrak{M}_{i}$ $=\left[\mathfrak{M}_{1}, \mathfrak{M}_{i-1}\right] \leqq\left[\mathfrak{M}_{1}, \mathfrak{M}_{1}+[L(\mathfrak{H}), R(\mathfrak{H})]\right]=\mathfrak{M}_{2}+\left[\mathfrak{M}_{1},[L(\mathfrak{R}), R(\mathfrak{H})]\right]$. Since the characteristic of $\mathfrak{F}$ is not two, it follows from (28) and (30) that $\left[L_{y},\left[L_{x}, R_{z}\right]\right]$ is in $\Omega_{0}$. Similarly it may be shown that $\left[R_{y},\left[L_{x}, R_{z}\right]\right]$ is in $\mathfrak{R}_{0}$. Hence $\mathfrak{M}_{i} \leqq \mathfrak{R}_{0}$ for all $i, \mathfrak{R} \leqq \mathfrak{R}_{0}$. But $\mathfrak{R}_{0} \leqq \mathfrak{M}_{1}+\mathfrak{M}_{2}$, so $\mathbb{R}=\mathfrak{R}_{0}$. It is clear from $(27)$ and $(28)$ that we may also write

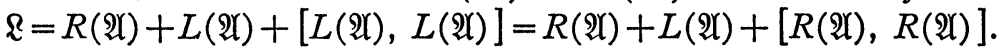

TheOREM 6. All derivations of a Cayley-Dickson algebra $\mathbb{E}$ over a field of characteristic zero are inner. They have the form (32). ${ }^{4}$

${ }^{4}$ Added in proof: Let $\mathfrak{A}$ be an alternative algebra with unity element over $\mathfrak{F}$ of characteristic not two or three. Then (using $\mathfrak{R}=R(\mathfrak{H})+L(\mathfrak{H})+[L(\mathfrak{H}), L(\mathfrak{H})]$ and (29)) one may prove that a derivation $D$ of $\mathfrak{A}$ is inner if and only if $D$ is the sum of two derivations: one of the form (32), the other of the form (15) with $d$ satisfying $[d, x, y]=0$ for all $x, y$ in $\mathfrak{A}$. For a Cayley-Dickson algebra $\mathbb{C}$ the second derivation in the sum is zero. 
The set of inner derivations of $\mathbb{E}$ is not $\{0\}$, for it is easy to choose a pair of quaternions $x, z$ in $\mathfrak{Q}$ such that $[z, x] \neq 0$. Then, applying $D$ in (31) to $v$ in (25), we obtain $v D=v[\bar{z}, \bar{x}]+v[z, \bar{x}]+v[z, x]=v[z, x]$ since $\bar{z}+z=t(z) 1$. Then $D \neq 0$. But $D$ is inner and, by the corollary to Theorem 3, all derivations of $\mathfrak{C}$ are inner, for it is known [3, p. 780] that the derivation algebra $\mathfrak{D}$ of $\mathfrak{E}$ is simple (the exceptional simple Lie algebra of dimension 14 ). To see that the set $\mathfrak{D}_{0}$ of derivations (32) is an ideal in $\mathfrak{D}$, it is sufficient to show that for $D_{1}$ in (29) we have $\left[D_{1}, D^{\prime}\right]$ in $\mathfrak{D}_{0}$ for any derivation $D^{\prime}$ of $\mathfrak{C}$. But this is an easy consequence of the Jacobi identity and the fact that $[x, z] D^{\prime}$ $=\left[x, z D^{\prime}\right]+\left[x D^{\prime}, z\right]$. Since $\mathfrak{D}_{0} \neq\{0\}, \mathfrak{D}_{0}=\mathfrak{D}$.

THEOREM 7. All derivations of a semi-simple alternative algebra $\mathfrak{A}$ over a field of characteristic zero are inner.

Since $\mathfrak{A}$ is the direct sum of simple components which are either associative or Cayley-Dickson algebras over their centers, and since it is well known that derivations of the associative components are inner, this theorem is reduced by Theorem 4 to the case where $\mathfrak{A}$ is a Cayley-Dickson algebra $\mathbb{S}$ over its center 3. But, since the characteristic is zero, the derivations of $\mathfrak{A}$ map the field $\mathbb{Z}$ upon $\{0\}$; moreover, if $\mathfrak{D}$ is the derivation algebra of $\mathfrak{E}$, then $\mathfrak{D}_{z}$ is the derivation algebra of $\mathfrak{A}$. Then by Theorem 6 all derivations of $\mathfrak{A}$ are inner.

4. Jordan algebras. A Jordan algebra is a commutative algebra $\mathfrak{Q}$ in which

$$
x^{2}(x y)=x\left(x^{2} y\right)
$$

for all $x, y$ in $\mathfrak{A}$.

A. A. Albert has shown [1, equation (8), p. 550] that in a Jordan algebra $\mathfrak{A}$ over $\mathfrak{F}$ of characteristic not two the identity

$$
\left[R_{y},\left[R_{x}, R_{z}\right]\right]=R_{(x y) z-x(y z)}
$$

holds. That is, $\left[R_{x}, R_{z}\right]$ is a derivation of $\mathfrak{A}$ for any $x, z$ in $\mathfrak{A}$. So is the sum

$$
D=\sum_{i}\left[R_{x_{i}}, R_{z_{i}}\right], \quad \text { for } x_{i}, z_{i} \text { in } \mathfrak{A} \text {. }
$$

THEOREM 8. The Lie transformation algebra of a Jordan algebra $\mathfrak{A}$ over a field of characteristic not two is

$$
\mathfrak{R}=R(\mathfrak{U})+[R(\mathfrak{I}), R(\mathfrak{U})] .
$$

If $\mathfrak{P}$ has a unity element 1 , then a derivation $D$ of $\mathfrak{A}$ is inner if and only if $D$ has the form (36). 
Since $\mathfrak{A}$ is commutative, $\mathfrak{M}_{1}=R(\mathfrak{U})$. Any element of $\mathfrak{M}_{3}=[R(\mathfrak{A})$, $[R(\mathfrak{H}), R(\mathfrak{H})]]$ is a sum of elements of the form (35), so $\mathfrak{M}_{3} \leqq \mathfrak{M}_{1}$. Then $\left[\mathfrak{M}_{2}, \mathfrak{M}_{2}\right] \leqq \mathfrak{M}_{4}=\left[\mathfrak{M}_{1}, \mathfrak{M}_{3}\right] \leqq\left[\mathfrak{M}_{1}, \mathfrak{M}_{1}\right]=\mathfrak{M}_{2}$ by (12). Hence $\mathfrak{M}_{1}+\mathfrak{M}_{2}$ is a Lie algebra, $\mathfrak{R}=\mathfrak{M}_{1}+\mathfrak{M}_{2}=R(\mathfrak{R})+[R(\mathfrak{H}), R(\mathfrak{H})]$. Let $D=R_{y}+\sum_{i}\left[R_{x_{i}}, R_{z_{i}}\right]$ be an inner derivation. Since $1 D=0$, we have $y=0$.

We refer the reader to the proof of the following theorem by $\mathrm{N}$. Jacobson in the paper mentioned in footnote 1.

Theorem 9 (Jacobson). All derivations of a semi-simple Jordan algebra $\mathfrak{A}$ over a field of characteristic zero are inner; they have the form (36).

\section{REFERENCES}

1. A. A. Albert, $A$ structure theory for Jordan algebras, Ann. of Math. vol. 48 (1947) pp. 546-567.

2. N. Jacobson, Abstract derivation and Lie algebras, Trans. Amer. Math. Soc. vol. 42 (1937) pp. 206-224.

3. - Cayley numbers and normal simple Lie algebras of type $G$, Duke Math. J. vol. 5 (1939) pp. 775-783.

4. R. D. Schafer, Alternative algebras over an arbitrary field, Bull. Amer. Math. Soc. vol. 49 (1943) pp. 549-555.

The Institute for Advanced Study 